\title{
Possible contrôle de la pigmentation cutanée chez l'Homme par des loci non homologues des loci de coloration classiques des Mammifères
}

\author{
J.P. CÉSARINI et Isabelle GUÉDON \\ I.N.S.E.R.M. U-212 \\ Fondation Rothschild, 25, rue Manin, 75019 Paris
}

\section{Résumé}

Les variations de taille des mélanosomes observées dans les mélanocytes de l'épiderme de l'Homme ne sont pas observées dans les mélanocytes pilaires. Ces variations de taille sont responsables de la digestion et de la disparition des mélanosomes dans les kératinocytes. Ce système semble ne pas avoir d'équivalent chez les autres Mammifères. En fonction des données de la physiopathologie, on peut alors concevoir des gènes supplémentaires contrôlant la couleur de l'épiderme, ses réactions pigmentées aux expositions U.V.

\section{Introduction}

Le rôle de la pigmentation du pelage des Mammifères est fort différent du rôle que joue la pigmentation cutanée chez l'Homme. Chez les Mammifères, les couleurs et leur répartition sur le corps trouvent leur justification dans les nécessités de reproduction (attraction sexuelle), de défense (lutte contre les prédateurs), ou de nutrition (camouflage, agressivité) (cf. ROUGEoT, 1981 pour revue). Le développement de l'intelligence chez l'Homme a réduit pratiquement à néant ces nécessités. Par contre, de nouvelles exigences se sont développées avec la disparition du pelage. Il s'agit essentiellement de la protection contre les rayonnements ultraviolets solaires et de la synthèse de vitamine D antirachitique par l'épiderme remplaçant l'apport lacté fortement raccourci (cf. Riquet, 1981 pour revue). La pression de sélection sur les gènes de la pigmentation est donc radicalement modifiée. Il est compréhensible alors que l'étude des variants aux loci homologues des Mammifères ait assez peu contribué à la compréhension des différences de pigmentations cutanées observées dans l'espèce humaine. Les différentes couleurs de cheveux, de poils axillaires et pubiens ne sont peut-être que la survivance des caractères pigmentaires ancestraux. C'est dans l'étude des variations de ces couleurs qu'il est possible de mettre en évidence les loci homologues des Mammifères. Cependant, la mobilité de l'Homme à la surface de la terre, proportionnelle au développement de son intelligence, a facilité les mélanges raciaux, rendant cette étude, déjà compliquée par l'absence de la couverture pileuse, encore plus difficile (cf. LAUVERGNE, 1981, pour revue). 
A côté de ces variants homologues qui ne se manifestent pas ou peu, l'évolution a certainement produit des variants d'autres types. Dans le présent article nous voudrions présenter quelques possibilités de leur mise en évidence.

\section{Originalité du système mélanocytaire humain (rappel)}

Dans les zones tégumentaires recouvertes de poils chez les Mammifères, les mélanocytes sont associés essentiellement aux bulbes pileux et la mélanine délivrée aux kératinocytes du poil teinte celui-ci dans la masse. Le renouvellement du poil se fait à partir d'un germe qui lui est associé. Il faut donc concevoir l'existence d'un réservoir de mélanocytes dans le derme capables de se multiplier à chaque cycle pilaire. L'épiderme entre les poils ne contient que peu de mélanocytes, généralement inactifs. C'est l'épaisseur des poils qui protège des radiations U.V. Dans l'espèce humaine, les mélanocytes intraépidermiques présents sur plus de $90 \%$ de la surface corporelle se sont particulièrement développés. Présents avant la naissance, ils ne se divisent que rarement et leur activité de synthèse de la mélanine dépend largement de l'exposition aux radiations solaires (directe ou par relais optiques et hormonaux). Il semble nécessaire, pour expliquer les variations de couleur du tégument entre les populations humaines, de prendre en compte l'activité des loci déjà existants pour les mélanocytes pilaires, ainsi que l'activité éventuelle d'autres loci spécifiques des mélanocytes intraépidermiques. Les variations de couleur de l'épiderme sont par ailleurs majorées par les expositions brutales aux rayons U.V., ce qui implique l'intervention d'une adaptation à l'environnement.

\section{Méthodes d'investigations}

Quatre méthodes permettent d'étudier le système mélanocytaire chez l'Homme.

1. - L'observation visuelle par comparaison à une gamme étalon de la couleur des cheveux * du tégument en hiver et après exposition prolongée au soleil. Examen en lumière de Wood (U.V. longs).

2. - L'examen de prélèvements cutanés au microscope optique et surtout électronique permettant de préciser le degré de la mélanogénèse, le type de mélanine formée, son mode de dégradation dans les kératinocytes.

3. - L'interrogatoire des sujets pour faire préciser la couleur des cheveux des ascendants, collatéraux, et descendants. Estimation du hâle solaire, du degré d'exposition et de la protection ainsi apportée.

4. - Le recensement des mutations et anomalies héréditaires portant sur la mélanogénèse en pathologie.

* Référencier Wella International. 


\section{Matériel d'étude}

Cent trente sujets atteints de tumeurs malignes cutanées ont été examinés et ont fait l'objet d'une enquête familiale. Des prélèvements de peau insolée et non insolée ont été effectués.

Trente sujets volontaires ont été soumis à la même enquête, et ont fait l'objet de biopsies cutanées en zone non insolée (face interne du bras à $10 \mathrm{~cm}$ du creux axillaire) et de prélèvements de cheveux.

Les données de la littérature portant sur les travaux effectués sur les races jaunes et noires ont été intégrés dans la discussion des résultats ainsi que les informations fournies par la pathologie héréditaire.

\section{Observations}

\section{1. - Les mélanosomes des cheveux}

Les mélanosomes des cheveux, quelles que soient leurs couleurs, sont toujours de taille supérieure à un micron. Ces mélanosomes sont en forme de ballon de rugby (eumélanosomes) chez les sujets caucasiens bruns ou blonds, mongoloïdes, négroïdes, ou sphériques (phaeomélanosomes) chez les sujets roux. Ils sont toujours transférés sur un mode isolé aux kératinocytes. Les mélanosomes sphériques sont issus d'une mélanogénèse particulière associée aux phaeomélanines.

\section{2. - Les mélanosomes de l'épiderme}

Il convient de considérer d'une part, les mélanosomes, produits de synthèse des mélanocytes, et d'autres parts, les modalités de transfert et de dégradation de ces mélanosomes dans les kératinocytes.

Les mélanocytes de la peau non insolée des sujets à cheveux roux synthétisent des phaeomélanosomes sphériques mesurant 0,5 micron. Ceux-ci sont rapidement dégradés après transfert dans les kératinocytes. Le tableau 1 montre la faible protection naturelle des sujets roux vis-à-vis des radiations ultraviolettes.

Les mélanocytes de la peau non insolée des sujets à cheveux blonds synthétisent les eumélanosomes très petits et en faible quantité. Transférés par groupes de 10 à 20 , ils disparaissent rapidement vers la $3^{\mathrm{e}}$ couche de l'épiderme.

Les mélanocytes des zones non insolées des sujets bruns caucasoïdes sont plus nombreux et ont une taille inférieure à un micron. On les trouve sous forme de mélanosomes complexes (5 à 15 mélanosomes) dans les kératinocytes où la rapidité de leur dégradation influence directement la couleur de l'épiderme du blanc au jaune. 


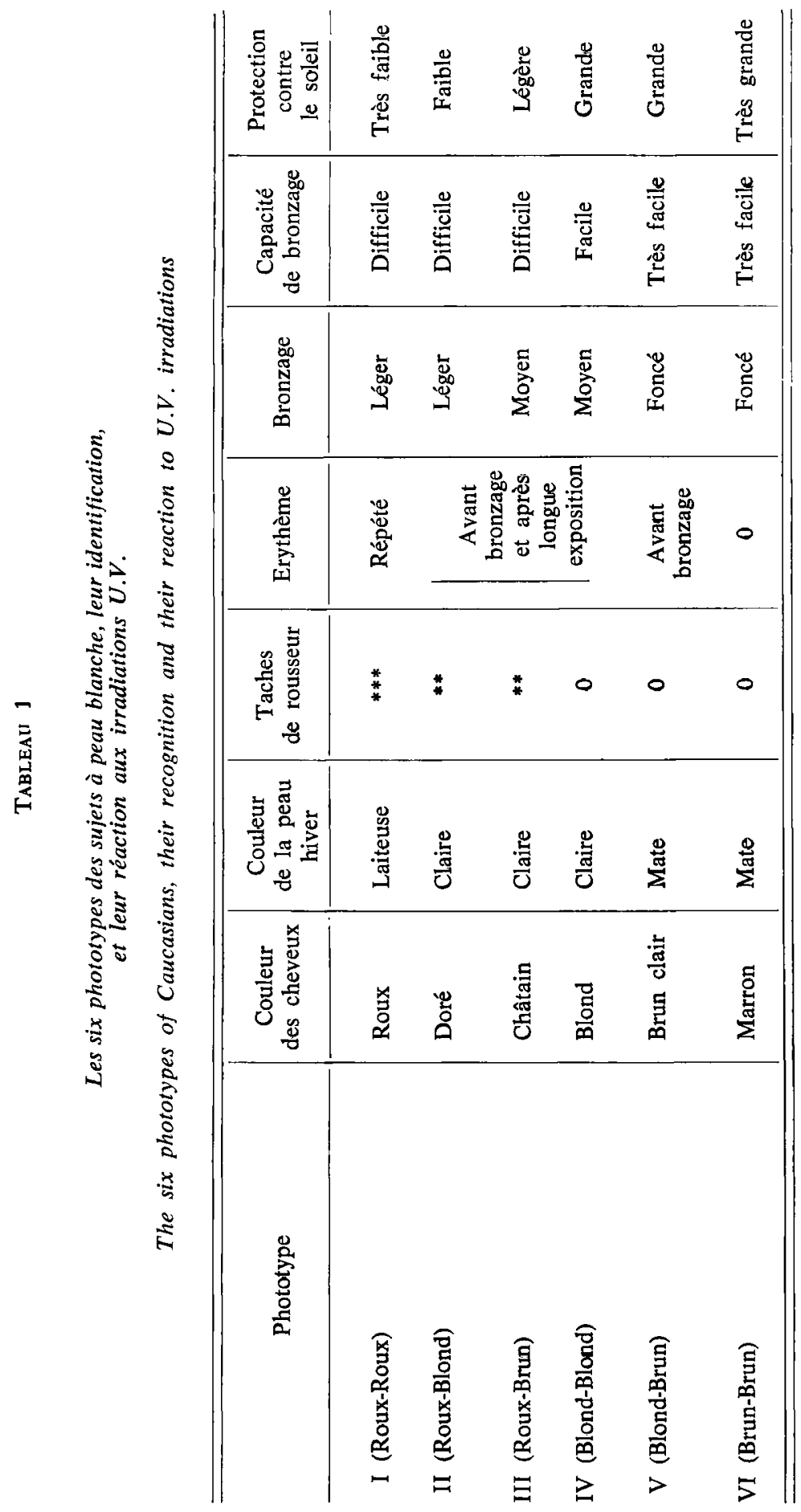


Chez les sujets négroïdes, les eumélanosomes sont de taille supérieure à un micron. Ils sont pour la plupart, transférés isolément aux kératinocytes où ils sont digérés lentement. Un bon nombre de ces mélanosomes est encore intact dans les couches cornées où ils absorbent la presque totalité de la lumière incidente. Les variations du nombre sont responsables des variations de teintes observées dans les populations négroïdes.

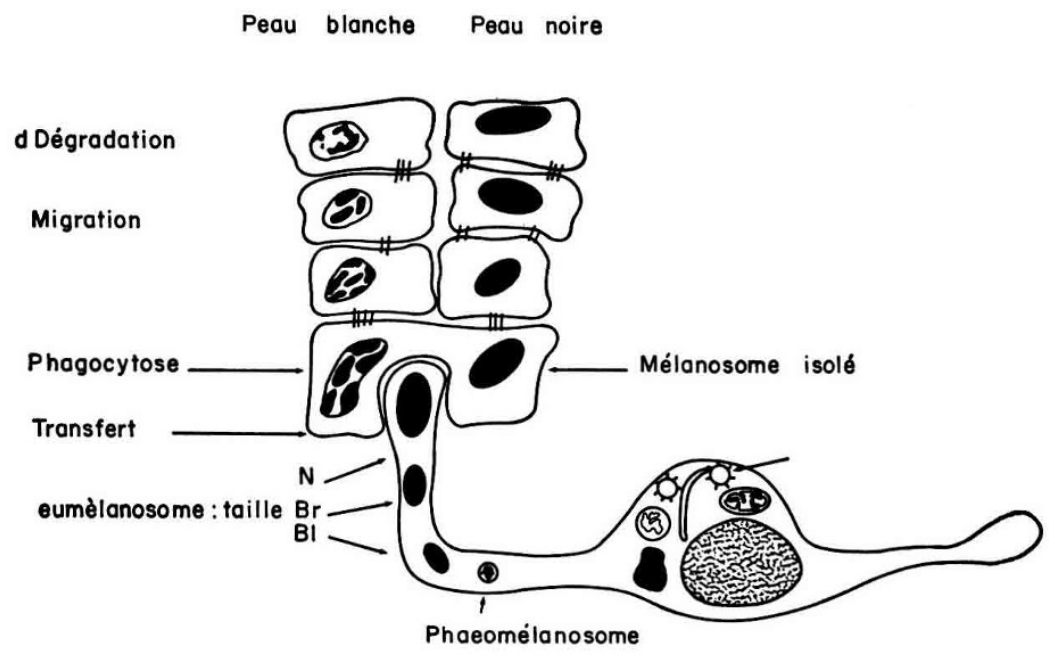

Fig. 1

Les différentes voies de synthèse et de dégradation des mélanosomes

The various pathways for the synthesis and degradation of melanosomes

Dans la figure 1 le schéma tente d'intégrer ces différentes informations auxquelles il faut ajouter quelques observations particulières. Un même mélanocyte peut présenter simultanément des eumélanosomes et des phaeomélanosomes, des eumélanosomes de petite et de grande taille (sujets métis caucasiens-négrö̈des). Les éphélides présentes uniquement sur les zones exposées chroniquement à la lumière des sujets à cheveux roux sont considérées comme des mutations induites par les U.V. Ce sont des îlots de mélanocytes synthétisant des eumélanines sur un fond de peau blanche à phaeomélanosomes.

Les eumélanosomes de petite taille sont remplacés par les mélanosomes de grande taille dans les mélanocytes exposés aux radiations U.V.A et $B$ en présence des psoralene (formation de ponts entre les deux brins de DNA). Les mélanomes malins, quelle que soit la couleur du sujet atteint, synthétisent des phaeomélanines, et les mélanocytes contiennent des phaeomélanosomes. 


\section{Analyse des résultats}

La forme des eumélanosomes semble être dictée par la présence de la matrice protéique eumélanique; ectte matrice n'étant pas formée si le mélanosome est orienté vers la production de phaeomélanines. [Les gènes Agouti $(A)$, Brun $(B)$, Albinisme $(C)$, Dilution $(D)$, et Extension $d u$ noir $(E)$ paraissent exister chez l'Homme, tant dans les poils que dans l'épiderme]. L'originalité du système pigmentaire de l'épiderme semble résider dans les variations de taille des mélanosomes, variations qui conditionnent à leur tour la dégradation plus ou moins rapide de ces mélanosomes. S'agit-il de trois allèles à un seul locus ou de plusieurs loci (système mendélien simple)? Un tel système pourrait être doublé par un système de variations polygéniques pour expliquer les différences quantitatives observées entre les mélanosomes de sujets blonds et bruns. Il semble qu'il y ait là une nouvelle voie de recherche, les conditions de dégradation de la mélanine devant être, par ailleurs, approfondies. Le tableau 1, bien que loin des réalités génétiques, correspond à la pratique quotidienne puisque plus de 98 p. 100 de la population à peau claire s'identifient facilement à l'un des 6 phototypes. La définition de sous-classes dans ces phototypes permettra peut-être d'identifier avec précision les gènes directeurs de la pigmentation cutanée.

Reçu pour publication en mars 1981.

\section{Summary}

Possible control of Human cutaneous pigmentation by loci which are not homologous with the classical color loci of Mammals

Epidermal melanocytes contain melanosomes the size of which varies. On the contrary, melanocytes of the hair bulbs contain remarkably regular sized melanosomes. Variations in size are responsible for the digestion and the disappearence of melanosomes in keratinocytes. This organization does not seem to have its counterpart in mammalians. According to physiopathological informations, one may conceive supplementary genes controlling the epidermal color and pigment darkning under sun exposures.

\section{Références bibliographiques}

CÉSARINI J.P., 1978. Réponses physiopathologiques du tégument humain aux radiations solaires. J. Med. Esth., 5, 61-70.

CÉSARINI J.P., 1979. Haarmelanin und haarfarbe. In : Haar und Haarkrankheiten, Von C.E. Orfanos. Fisher Verlag, Stuttgart N.Y., 137-166.

Lauvergne J.J., 1981. L'homologie entre l'Homme et les autres Mammifères pour les loci de colloration du pelage. Ann. Génét. Sél. anim., 13, (1) 57-66.

Nicholls E.M., 1968. Microspectrophotometry in the study of red hair. Ann. Hum. Genet., London, 32, 15-26.

Nicholls E.M., 1969. The genetic of red hair. Human Heredity, 19, 36-42.

Nicholls E.M., 1973. Pigment spotting in man and the number of genes determining skin and eye color. Human heredity, 23, 1-12.

Prota G., Searle A.G., 1978. Biochemical sites of gene action for melanogenesis in mammals. Ann. Genet. Sel. Anim., 10, 1-8. 
QueVEDo W.C., 1969. Genetic of mammalian pigmentation. In : The biologic effects of ultraviolet radiation. Ed. F. Urbach, Pergamon Press, London, N.Y., 315-324.

RiQuet R., 1981. Valeur adaptative de la pigmentation cutanée chez l'Homme. Ann. Génét. Sél. anim., 13, (1) 27-36.

Rougfot j., 1981. Téterminisne de la répartition de la pigmentation dans le pelage et la peau des Mammifères. Ann. Génét. Sél. anim., 13, (1) 9-16.

Sakurai T., Ochiai H., TakeUchi T., 1975. Ultrastructural change of melanosomes associated with Agouti pattern formation in mouse hair. Developmental Biology, 47, 466-471.

SEARLE A.G., 1968. Comparative genetics of coat colors in mammals. Logos Press-Academic Press, London, N.Y.

SEARLE A.G., 1975. Distribution and probable homologies of some genes determining coat color in mammals. In : Hand book of genetics, vol. 4, Plenum Press, 459-467.

Szabo G., Gerald A.B., Pathak M.A., Fitzpatrick T.B., 1972. The ultrastructure of racial color differences in man. In : Pigmentation : its genesis and biologic control. Ed. V. Riley, Appleton Century Crofts, N.Y., 23-41.

Takeuchi T., 1976. Genetic control of mammalian hair color. In : Biology and disease of the hair. Balt. Lond. Tokyo, University Park Press, 171-179.

Toda K., Pathak M.A., Parrish J.A., FitzPatrick T.B., Quevedo W.C., 1972. Alterations of racial differences in melanosome distribution in human epidermis after exposure to ultraviolet light. Nature New Biology, 236, 143-145.

Toda K., Matsumoto J., FitzPatrick T.B., 1976. Structure of melanosomes in human red hair bulbs. In : Biology and disease of the hair. Balt. Lond. Tokyo, University Park Press, 217-227.

Witkop C.S. Jr., White J.G., Nance W.E., Umber R.E., 1972. Mutations in the melanin pigment system in man resulting in features of oculocutaneous albinism. In : Pigmentations : its genesis and biologic control. Ed. V. Riley, Appleton Century Crofts, N.Y., 359-377. 